\title{
Role of Quantitative Fecal Immunochemical Test in Assessment of Mucosal Healing in Patients with Ulcerative Colitis
}

\author{
AHMAD F. ABO EL-EZZ AHMED, M.Sc.*; MOHAMED M. EL-BEDEWY, M.D.*; \\ MOHAMED A.S. ATTIA, M.D.** and MAHMOUD F. SELIM, M.D.* \\ The Departments of Internal Medicine* and Clinical Pathology**, Faculty of Medicine Tanta University, Egypt
}

\begin{abstract}
Background: Ulcerative Colitis (UC) is a chronic inflammatory disorder of the colorectum that is characterized by a remission and relapse course. Mucosal healing has been considered the goal of treatment in UC because it reduces the risk of relapse and colectomy. Fecal biomarkers have emerged as an important easy, rapid and non-invasive tool for assessing and monitoring mucosal healing in patients with UC.
\end{abstract}

Aim of the Study: To determine if the q-FIT can evaluate the mucosal healing in UC patients.

Patients and Methods: The study was conducted on 60 UC patients who underwent colonoscopy in the Endoscopy Unit, Internal Medicine Department in Tanta University Hospital. FIT was examined in stool samples from all the patients. Mucosal status was assessed using the Mayo Endoscopic Subscore (MES).

Data were collected from all study patients including; clinical, demographic and laboratory data. Statistical analysis was carried out for all collected data and statistical significance was determined at a $p$-value $<0.05$.

Results: A total of $60 \mathrm{UC}$ patients were evaluated with FIT results in conjunction with colonoscopies. The sensitivity and specificity of the FIT values $(<100 \mathrm{ng} / \mathrm{ml})$ for predicting MH (MES 0 alone) were 0.93 and 0.80 , respectively. The sensitivity and specificity when MH was defined as (MES 0 or 1) were 0.75 and 0.91 , respectively. A significant correlations between FIT levels and the MES was observed ( $r=0.858, p$ value $=0.001 *$ ).

Conclusion: Quantitative FIT can be a non-invasive and effectivebiomarker for evaluation of mucosal healing in UC.

Key Words: Ulcerative colitis - Mucosal healing - Quantitative faecal immunochemical test.

\section{Introduction}

ULCERATIVE Colitis (UC) is one of the 2 major types of Inflammatory Bowel Disease (IBD), along with Crohn's disease. It is a chronic inflammatory condition causing continuous mucosal inflammation

Correspondence to: Dr. Ahmad F. Abo El-Ezz Ahmed, E-Mail: ahmed fikry64@yahoo.com. of the rectum and the colon. It is characterized by a relapsing and remitting course [1] .

Patients with ulcerative colitis usually present with diarrhea, which may be associated with blood. Bowel movements are frequent and small in volume as a result of rectal inflammation. Associated symptoms include colicky abdominal pain, urgency, tenesmus, and incontinence [2] .

The treatment strategy for UC consists of remission induction therapy in the active stage, followed by maintenance of remission after successful remission induction. UC patients are treated to achieve and sustain clinical remission, as indicated by the disappearance of clinical symptoms. Recently, however, not only clinical remission but also endoscopic Mucosal Healing ( $\mathrm{MH})$ is being pursued as the treatment goal for UC [3-5] .

Endoscopically assessed mucosal healing is associated with improved outcomes for ulcerative colitis [6]. However, undergoing colonoscopy to determine mucosal healing is invasive and costly. Because clinical assessment in asymptomatic UC patients can underestimate mucosal disease activity, surrogate biomarkers of endoscopic disease assessment are appealing [7]. Therefore, non-invasive methods of evaluating and predicting mucosal status are eagerly desired.

Quantitative Fecal Immunochemical Tests (FITs) can measure hemoglobin concentrations in feces using an antibody for human hemoglobin. The amount of fecal blood most likely reflects mucosal status in UC. In particular, occult blood can be present in feces of UC patients in clinical remission but without mucosal healing. Such fecal occult blood can be detected by FIT, and therefore, a negative FIT result may reflect and predict mucosal healing non-invasively [8] 


\section{Patients and Methods}

This study was carried out over 60 Egyptian ulcerative colitis patients who underwent colonoscopy in the Endoscopy Unit at the Internal Medicine Department in Tanta University Hospital in the period from February 2016 to March 2017.

Mucosal status was assessed using the Mayo endoscopic subscore classification. It consists of four subscores: Stool frequency, rectal bleeding, endoscopic findings; and physician's global assessment. Each one of these subscores ranges from 0 to 3 , arriving at a final score between 0 and $12(\leq 2$ remission, 3-5 mild disease, 6-10 moderate disease, 11-12 severe disease).

\section{Inclusion criteria:}

All of the patients had an established diagnosis of UC according to endoscopic and histologic assessments.

\section{Exclusion criteria:}

- Patients who did not have FIT results within 1 month before colonoscopy.

- Patients with changes in abdominal symptoms or treatment after the FIT but before colonoscopy.

- If the colonoscopic examination was incomplete because of problems with the bowel preparation because the colonoscope could not be reached to the cecum.

\section{Method:}

All participants in this study were subjected to: Thorough history taking, full clinical examination, laboratory investigations in the form of: Full Blood Count, Erythrocyte Sedimentation Rate (ESR), C-Reactive Protein (CRP), serum albumin, quantitative fecal immunochemical test in stool and colonoscopy.

\section{Sampling and laboratory investigations:}

Sampling and all laboratory investigations were done in Clinical Pathology Department, Tanta University Hospitals. Fresh fecal sample is collected using Epitope Diagnostics Fecal Sample Collection Device. This test is intended for detection of fecal occult blood by the quantitative determination of human hemoglobin levels in stool samples by ELISA. The assay utilizes the two-site sandwich technique with two selected antibodies that bind to different epitopes of human hemoglobin. Assay standards, controls, and patient samples are added directly to wells of a microtiter plate that is coated with hemoglobin antibodies. Subsequently, a Horseradish Peroxidase (HRP)-conjugated human hemo- globin specific antibody is added to each well. After an incubation period, a sandwich of antibody human hemoglobin HRP-conjugated antibody is formed. The unbound antibody and buffer matrix is removed in the subsequent washing step. For the detection of this immune complex, the well is then incubated with a substrate solution in a timed reaction and then measured in a spectrophotometric microplate reader [9-11].

\section{Colonoscopy:}

The patients received a polyethylene glycol based electrolyte solution (e.g, moviprep or fortrans) for bowel preparation before colonoscopy. Total colonoscopy was done using an EPK-i5000 colonoscope (Pentax, Tokyo, Japan). Assessment of the mucosal status is done using the Mayo endoscopic subscore classification. Mucosal healing was defined as Mayo score ' 0 ' or ' 0 or 1' throughout the colorectum.

\section{Statistical analysis of the data:}

Data were fed to the computer and analyzed using IBM SPSS software package Version 20.0 (12). Quantitative data were described using range (minimum and maximum), mean, standard deviation and median. The distributions of quantitative variables were tested for normality using Kolmogorov-Smirnov test, Shapiro-Wilk test and D'Agstino test. If it reveals normal data distribution, parametric tests was applied. If the data were abnormally distributed, non-parametric tests were used. For normally distributed data, comparisons between more than two populations were analyzed F-test (ANOVA) to be used and Post Hoc test (LSD). For abnormally distributed data, Kruskal Wallis test was used to compare between different groups and pair wise comparison was assessed using Mann-Whitney test. Significance of the obtained results was judged at the $p$-value $<0.05$.

\section{Results}

In our study, age of the patients ranged from (17-68 years) with a mean age of $35.65 \pm 10.66$. There were 26 males $(43.3 \%)$ and 34 females $(56.7 \%)$.

As regard the clinical activity of the disease, there were $16(26.7 \%)$ patients in clinical remission and $44(73.3 \%)$ patients in clinically active disease.

Among the 16 patients in clinical remission, there were $12(75 \%)$ patients with MES 0, 3 (18.75\%) patients with MES 1, 1 (6.25\%) patient with MES 2 and no patients with MES 3. This means that the relation between clinical and endo- 
scopic remission was statistically significant ( $p$ value $=0.001)$ and that patients in clinical remission with MES 0 showed significantly lower FIT results than MES 1 and MES 2 ( $p$-value=0.017). (Table 1).

While comparing between the MES and platelets count, platelet counts were not related statistically to mucosal healing (by MES) ( $p$-value $=0.251$ ).

On the other hand, the comparison between the MES and TLC showed that there was statistically significant lower total leucocytic counts in patients with mucosal healing (by MES) than without ( $p$ value $=0.001)$. (Table 2 ).

On the other hand, the comparison between ESR results and MES were not statistically significant $(p$-value $=0.119)$. While there was statistically significant relation between MES and CRP values $(p$-value $=0.001) .($ Tables 3,4$)$.

The comparison between FIT results and colonoscopic findings by Mayo score showed the number of the cases with negative FIT (less than $100 \mathrm{ng} / \mathrm{ml}$ as cut-off value) was greatest in cases with Mayo 0 and gradually decreased as the activity of the disease increased. This means that patients in remission by Mayo score showed statistically significantly lower FIT results ( $p$-value $=0.001)$. (Table 5).

Our study has shown statistically significant positive correlation between FIT results and Mayo endoscopic score $(p$-value $=0.001 *)$ Fig. (1).

The diagnostic performance of FIT results in our patients when a negative FIT result was of less than $100 \mathrm{ng} / \mathrm{ml}$ as the cutoff value, showed that fecal immunochemical test results in relation to mucosal healing (Mayo endoscopic score 0 ) were $93 \%$ sensitivity and $80 \%$ specificity. Fig. (2).

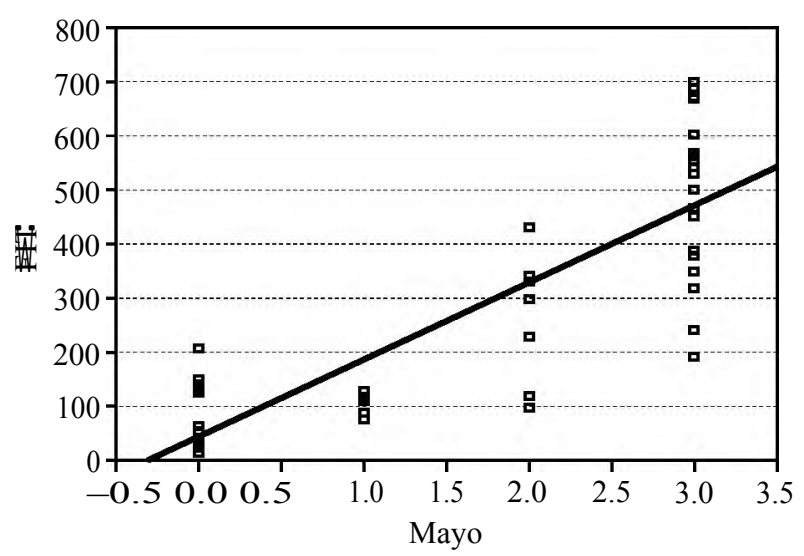

Fig. (1): Positive correlation between FIT results and Mayo endoscopic score.

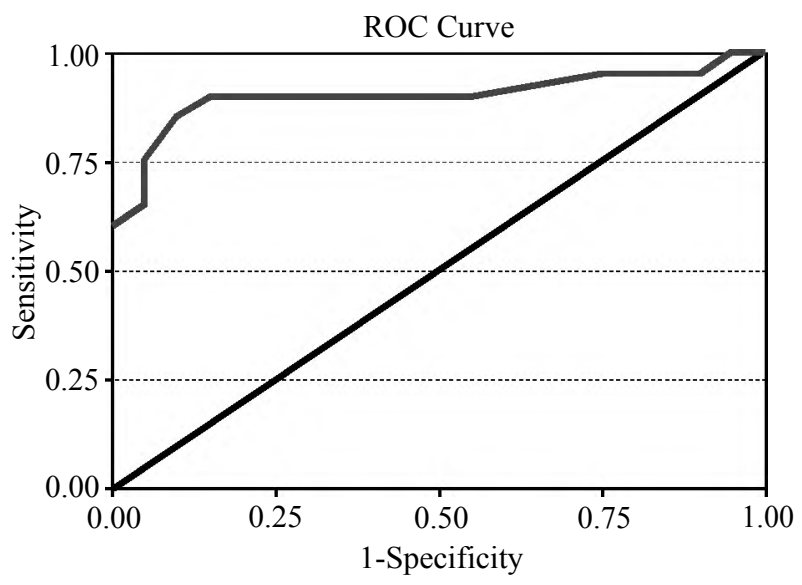

Fig. (2): Area under ROC curve denoting sensitivity of fecal immunochemical tests for mucosal healing (Mayo $0)$.

Table (1): Patients with clinical remission in comparison with MES.

\begin{tabular}{ll}
\hline Mayo & Clinical remission \\
\hline $0:$ & 12 \\
$\mathrm{~N}$ & $75.0 \%$ \\
$\%$ & 3 \\
$\mathrm{~N}$ & $18.75 \%$ \\
$\%$ & \\
& 1 \\
$\mathrm{~N}$ & $6.25 \%$ \\
$\%$ & \\
& \\
$\mathrm{~N}$ & 0 \\
$\%$ & $0 \%$ \\
Total: & \\
$\mathrm{N}$ & 16 \\
$\%$ & $100.0 \%$ \\
Chi-square: & \\
$\chi^{2}$ & 26.243 \\
$p$-value & $0.001 \%$ \\
\hline
\end{tabular}

$p$-value $=0.001 *$ (Significant).

Table (2): PLT and TLC values in comparison with MES.

\begin{tabular}{lllll}
\hline MES & Range & \multicolumn{1}{c}{ Mean \pm SD } & \multicolumn{1}{c}{$t$-test $\boldsymbol{p}$-value } \\
\hline$P L T X 10^{3} / \mathrm{ml}:$ & & & & \\
0 & $160-430$ & $276.611 \pm 89.361$ & 1.403 & 0.251 \\
1 & $210-330$ & $272.429 \pm 44.436$ & & \\
2 & $190-380$ & $283.666 \pm 74.353$ & & \\
3 & $160-500$ & $325.423 \pm 102.617$ & & \\
$T L C X 10^{3} / \mathrm{ml}:$ & & & & \\
0 & $4-10.8$ & $6.984 \pm 2.144$ & 5.878 & $0.001^{*}$ \\
1 & $4.5-12.9$ & $7.885 \pm 2.775$ & & \\
2 & $3.9-10.9$ & $7.533 \pm 2.374$ & & \\
3 & $4.2-16.9$ & $10.355 \pm 3.334$ & & \\
\hline
\end{tabular}


Table (3): Comparison between ESR and MES of the studied patients.

\begin{tabular}{lllll}
\hline Mayo & Range & Mean \pm SD & $t$-test & $p$-value \\
\hline ESR $(\mathrm{mm} / \mathrm{h}):$ & & & & \\
MES0 & $5-140$ & $38.56 \pm 36.42$ & 2.038 & 0.119 \\
MES 1 & $10-40$ & $22.86 \pm 11.13$ & & \\
MES2 & $20-95$ & $45.56 \pm 27.44$ & & \\
MES3 & $10-145$ & $53.08 \pm 31.02$ & & \\
\hline
\end{tabular}

Table (4): Comparison between CRP and MES of the studied patients.

\begin{tabular}{|c|c|c|c|c|}
\hline \multirow{2}{*}{ Mayo } & \multicolumn{3}{|c|}{ CRP } & \multirow{2}{*}{ Total } \\
\hline & Pos. & & Neg. & \\
\hline \multicolumn{5}{|l|}{$0-1$} \\
\hline $\mathrm{N}$ & 8 & & 17 & 25 \\
\hline$\%$ & $32 \%$ & $68 \%$ & 61 & $00.0 \% / 0$ \\
\hline \multicolumn{5}{|l|}{ 2-3: } \\
\hline $\mathrm{N}$ & 26 & & 9 & 35 \\
\hline$\%$ & $74.3 \%$ & & $25.7 \%$ & $100.0 \%$ \\
\hline \multicolumn{5}{|l|}{ Total: } \\
\hline $\mathrm{N}$ & 34 & & 26 & 60 \\
\hline$\%$ & $56.7 \%$ & & $43.3 \%$ & $100.0 \%$ \\
\hline \multicolumn{5}{|c|}{ Chi-square: } \\
\hline$x^{2}$ & & 10.623 & & \\
\hline$p$-value & & $0.001 *$ & & \\
\hline
\end{tabular}

Table (5): Comparison between FIT results and colonoscopic findings.

\begin{tabular}{|c|c|c|c|}
\hline \multirow{2}{*}{$\begin{array}{l}\text { Mayo } \\
\text { subendoscopic } \\
\text { score }(0-3)\end{array}$} & \multicolumn{2}{|c|}{ FIT } & \multirow{2}{*}{ Total } \\
\hline & Positive & Negative & \\
\hline \multicolumn{4}{|l|}{$0:$} \\
\hline $\mathrm{N}$ & 6 & 12 & 18 \\
\hline$\%$ & $33.3 \%$ & $66.7 \%$ & $100.0 \%$ \\
\hline \multicolumn{4}{|l|}{ 1: } \\
\hline $\mathrm{N}$ & 5 & 2 & 7 \\
\hline$\%$ & $71.4 \%$ & $28.6 \%$ & $100.0 \%$ \\
\hline \multicolumn{4}{|l|}{ 2: } \\
\hline $\mathrm{N}$ & 8 & 1 & 9 \\
\hline$\%$ & $88.9 \%$ & $11.1 \%$ & $100.0 \%$ \\
\hline \multicolumn{4}{|l|}{ 3: } \\
\hline $\mathrm{N}$ & 26 & 0 & 26 \\
\hline$\%$ & $100.0 \%$ & $0 \%$ & $100.0 \%$ \\
\hline \multicolumn{4}{|l|}{ Total: } \\
\hline $\mathrm{N}$ & 45 & 15 & 60 \\
\hline$\%$ & $75.0 \%$ & $25.0 \%$ & $100.0 \%$ \\
\hline \multicolumn{4}{|l|}{ Chi-square: } \\
\hline$x^{2}$ & \multicolumn{2}{|c|}{26.307} & \\
\hline$p$-value & \multicolumn{2}{|c|}{$0.001 *$} & \\
\hline
\end{tabular}

\section{Discussion}

Ulcerative Colitis (UC) is a chronic inflammatory disorder of the colorectum that is characterized by a clinical course of remission and relapse. Clinical remission is defined as a Mayo stool frequency of 0 or 1 and a Mayo rectal bleeding subscore of 0 [13]. The clinical status of the disease does not always correlate with the mucosal status, so continued monitoring of clinical manifestations as well as mucosal healing is required [14] .

The non-invasive, low cost, and rapid FIT is applied to assess the mucosal status [15]. Q-FIT uses antibodies directed against human hemoglobin [16].

The present study was conducted in the Endoscopy Unit at the Department of Internal Medicine in Tanta University Hospital and was carried out on 60 UC patients with variable degrees of activity according to Mayo score.

In our study, there was a slight female predominance as females represented $56.7 \%$ and males represented $43.3 \%$ of the patients. Nakari et al., [15] who studied the evaluation of mucosal healing of UC by FIT, documented that $49 \%$ of the patients were females and $51 \%$ were males.

Regarding CRP, our study showed that the increase in the mayo endoscopic score is associated with elevation in CRP values and it was statistically significant $(p$-value $=0.001)$. This was in agreement with Solem et al., [17] who discussed the correlation of CRP with clinical, endoscopic, histologic, and radiographic activity in IBD.

Against to our study, Auzoux et al., [18] who studied the assessment of mucosal healing in inflammatory bowel disease, indicated that the increase in CRP is not specific to inflammatory illness and can also be observed in cases of sepsis or systemic inflammation.

As regard ESR, there was statistically nonsignificant comparison with mucosal healing according to MES in our study ( $p$-value $=0.063$ ). This was in agreement with Lok et al., [19] who discussed the correlation between ESR and mucosal inflammation in Chinese ulcerative colitis patients.

Another study by Yoon et al., [20] who discussed the correlations of CRP and ESR with endoscopic activity indices in patients with ulcerative colitis, showed that ESR level were modestly correlated with endoscopic activity indices, including Mayo score, in UC patients with low sensitivity and specificity for detecting endoscopic remission. 
This discrepancy between the ESR and CRP results in assessment of the mucosal status may be explained by the application of these markers in systemic inflammation and so, they are non-specific to inflammation of the mucosa. Shinzaki et al., [21] studied the Leucine-rich Alpha-2 Glycoprotein as a novel serum biomarker for detecting mucosal healing during disease course in UC patients with normal CRP, explained that the inflammation is regulated mainly by cytokine[s] other than IL-6 that stimulates the synthesis of CRP in the liver.

Based on the results of our study, endoscopic remission was significantly corresponding to clinical remission as $75 \%$ of patients in clinical remission were MES 0 and $18.75 \%$ of them were MES 1. This was in agreement with Lewis et al., [22] who used the non-invasive components of Mayo score (the stool frequency and rectal bleeding) to assess the clinical response in UC patients.

Opposite to our study, Meucci et al., [23] who studied a group of mild to moderate UC patients, demonstrated that there was no correlation between the rate of clinical remission and the degree of endoscopic activity.

In our study, MES 0 patients in clinical remission presented significantly lower FIT results than MES 1-3. This was in line with the results of Nakari et al., [24] who studied the correlation between FIT results, mucosal healing and risk of relapse in UC patients in clinical remission.

In the same context, Ryu et al., [25] demonstrated that fecal hemoglobin can be used as a marker of clinical disease activity in patients with UC, with a negative FIT accurately reflecting disease remission with the advantage of easy availability, fast results and low financial cost.

However, Jauregui-Amezaga et al., [26] reported that $27 \%$ of UC patients in clinical remission with mucosal healing relapsed within one year. This is attributed to the natural course of UC. Considering such fact, non invasive biomarkers that assess and indicate the continuation of remission would be relevant.

Consequently, a study performed by Hiraoka et al., [27] concluded that throughout the followup of UC patients who were confirmed to have MH with negative FIT, clinical remission continued as long as FIT was negative, and that positive conversion of the FIT, particularly with high values, could be correlated with clinical relapse a few months later.
In this study, the predictability of $\mathrm{MH}$ was compared by FIT results. FIT results and colonoscopic findings by Mayo score showed statistically significant positive correlation in assessment of mucosal healing as $p$-value $=0.001$. This was in agreement with Nakari et al., [15] and Ryu et al., [25] who reported statistically significant positive correlation between fecal hemoglobin concentrations and MES ( $p$-value $<0.01$ and $<0.0001$ respectively).

\section{Conclusion:}

These findings suggest that quantitative fecal immunochemical test was reliable, non-invasive, easy, rapid and low cost tool to reflect the colonic mucosal status in patients with UC. Therefore, a negative FIT was strongly correlated with mucosal healing, and so quantitative FIT can be considered a novel marker for ulcerative colitis diagnosis and follow-up.

\section{Recommendations:}

Future studies including larger number of patients and longer duration are required to better clarify the exact role of FIT in assessment of mucosal healing in UC patients, monitoring of the disease activity and to inform clinical decisions to modify the treatment.

\section{Acknowledgements:}

We would like to thank all participants who helped during this study.

\section{Conflict of interest:}

None declared.

\section{References}

1- DIGNASS A., ELIAKIM R., MAGRO F., et al.: Second European evidence-based consensus on the diagnosis and management of ulcerative colitis part 1: Definitions and diagnosis. J. Crohns. Colitis., 6 (10): 965-90, 2012.

2- SILVERBERG M.S., SATSANGI J., AHMAD T., et al.: Toward an integrated clinical, molecular and serological classification of inflammatory bowel disease: Report of a Working Party of the 2005 Montreal World Congress of Gastroenterology. Can. J. Gastroenterol., 19 Suppl A: $5 \mathrm{~A}, 2005$.

3- PEYRIN-BIROULET L. et al.: Results from the ${ }^{2 \text { nd }}$ Scientific Workshop of the ECCO. I: Impact of mucosal healing on the course of inflammatory bowel disease. J. Crohns. Colitis., 5: 47783, 2011.

4- LOPEZ-PALACIOS N., MENDOZA J.L., TAXONERA C., et al.: Mucosal healing for predictingclinical outcome in patients with ulcerative colitis using thiopurines in monotherapy. Eur. J. Intern. Med., 22: 621-5, 2011. 
5- CHEN L.S., YEN A.M., CHIU S.Y., et al.: Baseline faecal occult blood concentrationas a predictor of incident colorectal neoplasia: Longitudinal follow-up of a Taiwanese population-based colorectal cancer screening cohort. Lancet Oncol., 12: 551-8, 2011.

6- FROSLIE K.F., JAHNSEN J., MOUM B.A., et al.: Mucosal healing in inflammatory bowel disease: Results from a Norwegian population-based cohort. Gastroenterology, 133: 412-22, 2007.

7- LEWIS J.D.: The utility of biomarkers in the diagnosis and therapy of inflammatory bowel disease. Gastroenterology, 140: 1817-26, 2011.

8- KATO J., HIRAOKA S., NAKARAI A., et al.: Fecal immunochemical test as a biomarker for inflammatory bowel diseases: Can it rival fecal calprotectin?, 14 (1): 5-14, 2016.

9- SIEG A., HERTEL A., JOHN M.R., et al.: Screening for colorectal neoplasms with a new immunological human faecalhaemoglobin and albumin test. Eur. J. Cancer Prev., 7 (4): 279-85, 1998.

10- LEVI Z., ROZEN P., HAZAZI R., et al.: A quantitative immunochemical fecal occult blood test for colorectal neoplasia. Ann. Intern. Med., 146 (4): 244-55, 2007.

11- FRASER C.G., MATTHEW C.M., MOWAT N.A., et al.: Immunochemical testing of individuals positive for guaiac faecal occult blood test in a screening programme for colorectal cancer: An observational study. Lancet Oncol., 7 (2): 127-31, 2006.

12- VERMA J.: Statistics Computer programs. In: A simple guide to IBM SPSS statistics for version 20.0.By: Kirkpatrick A. and Feeney C. Student Ed. Ch4 th. Belmont, Calif.: Wadsworth, Cengage Learning, p.: 80-100, 2013.

13- COLOMBEL J.F., RUTGEERTS P., REINISCH W., et al.: Early mucosal healing with infliximab is associated with improved long-term clinical outcomes in ulcerative colitis. Gastroenterology, 141 (4): 1194-201, 2011.

14- MENEES S., HIGGINS P., KORSNES S., et al.: Does colonoscopy cause increased ulcerative colitis symptoms? Inflamm. Bowel. Dis., 13: 12-8, 2007.

15-NAKARAI A., KATO J., HIRAOKA S., et al.: Evaluation of mucosal healing of ulcerative colitis by a quantitative fecal immunochemical test. Am. J. Gastroenterol., 108: 83-9, 2013.

16- VILKIN A., ROZEN P., LEVI Z., et al.: Performance characteristics and evaluation of an automated-developed and quantitative, immunochemical, fecal occult blood screening test. Am. J. Gastroenterol., 100: 2519-25, 2005.

17- SOLEM C.A., LOFTUS E.V., TREMAINE W.J., et al.:
Correlation of C-reactive protein with clinical, endoscopic, histologic, and radiographic activity in inflammatory bowel disease. Inflamm. Bowel. Dis., 11 (8): 707-12, 2005.

18- AUZOUX J., BOSCHETTI G., LAHLOU W., et al.: Assessment of Mucosal Healing in Inflammatory Bowel Disease. J. Clin. Gastroenterol. Hepatol., 1: 1, 2016.

19- LOK K.H., NG C.H., HUNG H.G., et al.: Correlation of serum biomarkers with clinical severity and mucosal inflammation in Chinese ulcerative colitis patients. J. Dig. Dis., 9: 219-24, 2008.

20- YOON J.Y., PARK S.J., HONG S.P., et al.: Correlations of C-reactive protein levels and erythrocyte sedimentation rates with endoscopic activity indices in patients with ulcerative colitis. Dig. Dis. Sci., 59: 829-37, 2014.

21- SHINZAKI S., MATSUOKA K., IIJIMA H., et al.: Leucine-rich Alpha-2 Glycoprotein is a SerumBiomarker of Mucosal Healing in Ulcerative Colitis. J. Crohns. Colitis., 84-91, 2017.

22- LEWIS J.D., CHUAI S., NESSEL L., et al.: Use of the noninvasive components of the Mayo score to assess clinical response in ulcerative colitis. Inflamm. Bowel. Dis., 14: 1660-6, 2008.

23- MEUCCI G., FASOLI R., SAIBENI S., et al.: Prognostic significance of endoscopic remission in patients with active ulcerative colitis treated with oral and topical mesalazine: A prospective, multicenter study. Inflamm. Bowel. Dis., 18 (6): 1006-10, 2012.

24- NAKARAI A., KATO J., HIRAOKA S., et al.: Ulcerative colitis patients in clinical remission demonstrate correlations between fecal immunochemical test results, mucosal healing, and risk of relapse World J. Gastroenterol., 22 (21): 5079-87, 2016.

25- RYU D.G., KIM H.W., PARK S.B., et al.: Assessment of disease activity by fecal immunochemical test in ulcerative colitis. World J. Gastroenterol., 22 (48): 10617-24, 2016.

26- JAUREGUI-AMEZAGA A., LOPEZ-CERON M., ACEITUNO M., et al.: Accuracy of advanced endoscopy and fecal calprotectin for prediction of relapse in ulcerative colitis: A prospective study. Inflamm. Bowel. Dis., 20: 1187-93, 2014

27- HIRAOKA S., KATO J., NAKARAI A., et al.: Consecutive Measurements by Faecal Immunochemical Test in Quiescent Ulcerative Colitis Patients Can Detect Clinical Relapse. J. Crohns. Colitis., 687-94, 2016. 


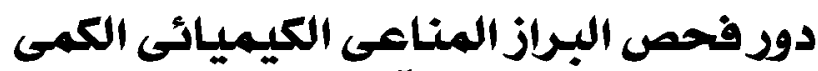

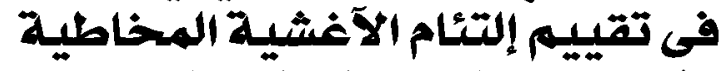

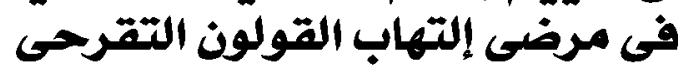

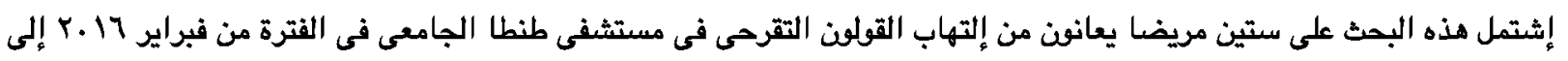

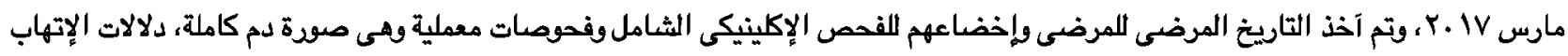

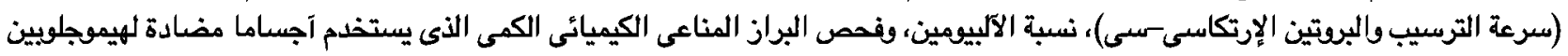

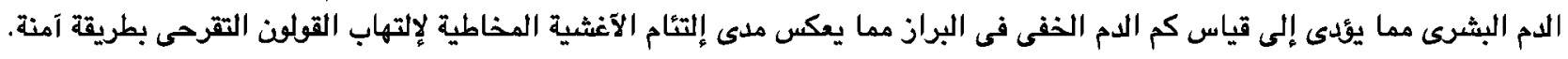

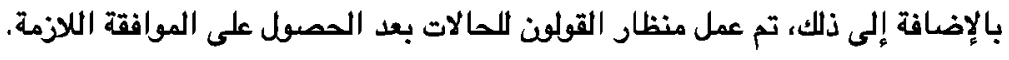
وقد خلصت هذه الدراسة إلى إنخفاض ذو قيمة إحصائية بمستويات فحص البراز المناعى الكمى فى مرضى إلتهاب القولون التقرحى فى

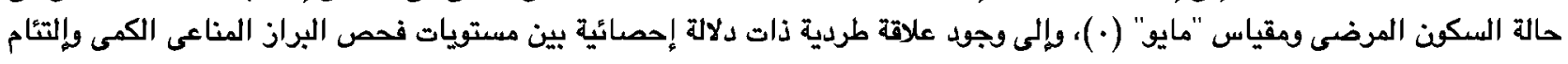

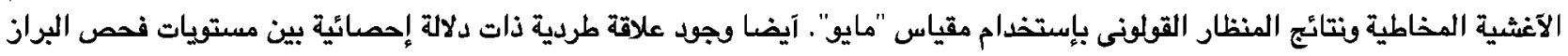
المناعى الكمى ونتائج مقياس "مايو". 